\section{(Zebra)fishing for drugs}

\section{By Michael J. Haas, Senior Writer}

North American researchers have developed a high throughput screening method that relies on zebrafish behavior to identify new neuroactive molecules and scaffolds. ${ }^{1}$ The technology could streamline drug discovery by providing initial in vivo data about neuroactive drug candidates earlier and more cheaply than the rodent models used in preclinical development.

Most compound screens use in vitro assays that reproduce only a few of the interactions that occur in whole organisms. This lack of complexity is especially challenging to drug discovery in neurology because in vitro assays cannot model a compound's effect on behavior and brain activity. Meanwhile, studies in the standard neurological rodent models can be long and expensive-factors that preclude their use in upstream drug discovery.

Zebrafish (Danio rerio) have long been used as models to study development and gene function in vertebrates. But Randall Peterson and colleagues hit upon the idea of using them to model responses to small molecules after discovering the fish exhibited very specific behaviors in response to light stimulus.

Peterson is an assistant professor of medicine at Harvard Medical School and Massachusetts General Hospital (MGH) and an associate member of the Broad Institute of MIT and Harvard.

David Kokel, a postdoctoral research fellow in Peterson's MGH group, found that zebrafish embryos moved upon exposure to light. "He was probably not the first person ever to notice this," but he was intrigued by the behavior because zebrafish embryos don't have photoreceptors in their eyes, Peterson said.

The team found that the embryos exhibited regular and predictable motor responses to a 30-second pulse of light, which the group suspected could be the basis for a behavior-based screen. They developed a 14-part barcode that represented the intensity of embryos' photomotor responses during different time periods across the 30-second pulse.

The barcode for embryos exposed only to the light pulse provided a baseline of normal behavior as a control for embryos used in subsequent screens of library compounds.

The researchers screened a library of 14,000 small molecules against the embryos' photomotor responses to a 30 -second pulse and compared the resulting barcodes with those for controls. They found that the small molecule-induced barcodes fell into many distinct clusters, each corresponding to a particular phenotype in the zebrafish.

For known neuroactive compounds, the clusters corresponded to the mode of action of the compounds, including targets and pathways. For example, barcodes for dopamine receptor agonists indicated intense photomotor activity throughout the second half of the pulse, whereas barcodes for adrenergic receptor agonists indicated intense photomotor activity at the beginning and end of the pulse.

Given the results for known compounds, the team suggested the barcode clusters could serve as starting points for identifying the targets of new molecules. To test this hypothesis, they selected two compounds not previously known to be neuroactive but whose barcodes clustered with several acetylcholinesterase (AChE) inhibitors. In vitro and zebrafish assays confirmed that the compounds were previously unknown AChE inhibitors.

Combining barcode information with structural features also enabled the researchers to identify the targets of new compounds. As an example, the team found correlations in the barcodes of several structurally related coumarins. Computational modeling predicted that the shared structure could bind monoamine oxidases and in vitro assays confirmed the coumarins as previously unknown inhibitors of monoamine oxidase A (MAO-A) or MAO-B.

Data were reported in Nature Chemical Biology, and the team included scientists from the Stanley Center for Psychiatric Research at the Broad Institute, The University of British Columbia and the University of California, San Francisco.

"The study demonstrates that simple evoked behavioral endpoints may be monitored, be standardized and - with high throughput-provide a technique that may be powerful enough to address putative new targets and pathways for the treatment of psychiatric disorders," said Lars Rønn, head of CNS pharmacology-neurology at NeuroSearch A/S.

Karin Troelsen, a research scientist in CNS pharmacology-neurology at NeuroSearch, noted that some compounds produced such severe reductions in embryo photomotor activity that Peterson's team labeled them as toxic. Thus, she suggested the technology could be used to screen compounds for toxicity as well as neuroactivity.

Adrian Hill, associate director at Evotec AG, told SciBX the screen "appears to provide a relatively high information content, and on the basis of a first-pass reprofiling screen, that could allude to whether a compound was acting either directly on, upstream or downstream to a known target."

More importantly, he added, the screen could show whether a compound was potentially acting "via an unknown pathway which could lead to the discovery of novel therapeutics."

Evotec uses zebrafish to identify therapeutic candidates, screen client compounds for safety and toxicity liabilities and develop 
various disease models including those for CNS disorders.

NeuroSearch uses a range of techniques to identify and develop compounds to treat psychiatric disorders, pain and other neurological disorders. The company's Integrative Screening Process (ISP) maps the treatment response of a whole organism.

Hill and Rønn both said the main advantage of the technology was the ability to test many compounds in vivo early in the drug discovery process.

Hill noted that the small size of zebrafish makes them compatible with multiple components of high throughput screens, including equipment and concentrations of compound libraries typically associated with in vitro cell-based models. "In contrast to the zebrafish, most other in vivo studies in drug discovery come much later in the process, as compound scale-up is generally required in order to adequately dose the larger mass of the animals," he said.

Getting in vivo data before the point of traditional compound scale-up "could allow you to better prioritize compound hits based on activity, toxicity and safety and thus reduce attrition" later in the development process, said Hill. "The technology could also be used to investigate specific targets or phenotypes, including those generated by chemical insult, genetic mutation or morpholino knockdown. So they have a way to focus this assay, in addition to doing broadbased screens."

Added Rønn: "It will be exciting to follow the application of this methodology to more advanced behavioral endpoints and to the analysis of disease-related behavioral dysfunction induced pharmacologically or by genetic manipulation.”

\section{Screening business}

Despite the advantages of early in vivo screening, dosing parameters and the inherent properties of zebrafish embryos could limit the utility of the technology.

The method "may not be able to provide all the answers in a single assay, and follow-up studies will always be needed for confirmation," said Hill.

He noted that some of the false negatives and toxic compounds found by Peterson's team could have resulted from dosing limitations.

"Dosing has to be limited in a high throughput screen like this, but as the team only tested each compound at concentration(s) between 10 and $100 \mu \mathrm{M}$, that might not be suitable for all compounds to see optimal activity," he said.

In addition, Hill told SciBX that many factors determine the uptake of a compound by the zebrafish when treated in this way. If such factors had been taken into account with a bioanalysis that measured the amount of compound absorption "they might have had fewer false negatives and/or toxicities; but this would be impractical in such a screen," he said.

Regardless, said Hill, "secondary screens can always be used to confirm and check the first-pass results. When one considers the alternatives, an in vivo profiling tool in this high throughput screen format that can identify drugs which alter neuroactivity has to be viewed as a significant advancement for this field."

Peterson agreed that testing compounds at multiple doses could be useful for some screening applications.

"In a more careful dose-response study, we have seen varying effects at higher or lower concentrations," he said. "So for discovery efforts, where screening tens of thousands of compounds is the goal, it may be desirable to screen at a single dose. Other studies looking at smaller numbers of compounds could certainly be done at multiple doses to increase the likelihood that the compounds' effects are detected."

Troelsen and Rønn noted that the screening method might be limited by characteristics of the zebrafish embryos.

For example, Troelsen said the adrenergic, dopaminergic and serotonergic pathways appeared to play key roles in the embryos' photomotor responses. "This might make it hard to identify compounds that have novel mechanisms of actions, unless those mechanisms somehow interact with a pathway in the photomotor response," she said.

Troelsen also noted that the screen could not distinguish between compounds that act on the zebrafish CNS from those that might be acting on the peripheral nerves or muscles. She added that the brain is not fully developed and the blood brain barrier (BBB) is not present in zebrafish embryos, including those used by Peterson's team. Thus, she cautioned that a compound's activity in those embryos might not be the same as it is in humans.

Peterson said his team plans to develop new types of automated behavioral assays. Ongoing work includes testing the inhibitors identified in the screen and conducting screens to identify compounds that modify genetic and pharmacologically induced disease phenotypes in the zebrafish.

The findings reported in Nature Chemical Biology are patented by $\mathrm{MGH}$ and are available for licensing.

Haas, M.J. SciBX 3(5); doi:10.1038/scibx.2010.139

Published online Feb. 4, 2010

\section{REFERENCES}

1. Kokel, D. et al. Nat. Chem. Biol.; published online Jan. 17, 2010; doi:10.1038/nchembio.307

Contact: Randall T. Peterson, Harvard Medical School, Charlestown, Mass.

e-mail: peterson@cvrc.mgh.harvard.edu

Contact: David Kokel, same affiliation as above e-mail: dkokel@cvrc.mgh.harvard.edu

\section{COMPANIES AND INSTITUTIONS MENTIONED}

Broad Institute of MIT and Harvard, Cambridge, Mass.

Evotec AG (Xetra:EVT), Hamburg, Germany

Harvard Medical School, Boston, Mass.

Massachusetts General Hospital, Boston, Mass.

NeuroSearch A/S (CSE:NEUR), Ballerup, Denmark

Stanley Center for Psychiatric Research at the Broad Institute,

Cambridge, Mass.

The University of British Columbia, Vancouver, British Columbia, Canada

University of California, San Francisco, Calif. 